\title{
Aspectos florísticos, fitossociológicos e ecológicos de um sub-bosque de terra firme na Amazônia Central, Amazonas, Brasil. ${ }^{1}$
}

\author{
Arlem Nascimento de OLIVEIRA², Iêda Leão do AMARAL ${ }^{3}$
}

\begin{abstract}
RESUMO
Os ecossistemas amazônicos caracterizam-se pela alta diversidade de espécies. Foram realizadas as análises florísticas e fitossociológicas de 0,05 ha de um sub-bosque na Amazônia Central (60¹2'40" W e 2³5'45" S), adotando-se o critério de distribuição em classes de altura: $\mathrm{C} 1$ (altura $\leqslant 0,5 \mathrm{~m}), \mathrm{C} 2(0,5<$ altura $\leqslant 1,5 \mathrm{~m}), \mathrm{C} 3(1,5<$ altura $\leqslant 3,0 \mathrm{~m})$ e C4 (altura $>3 \mathrm{~m} \mathrm{e} \mathrm{CAP}$ $<0,3 \mathrm{~m}$ ). Foram levantados 2.434 indivíduos, pertencentes a 67 famílias, 163 gêneros e 355 espécies. Fabaceae (27), Mimosaceae (22), Lauraceae (21), Caesalpiniaceae e Rubiaceae (18) constituem as cinco famílias mais ricas em espécies. Quanto ao número de indivíduos, as famílias Marantaceae (209), Chrysobalanaceae (198), Mimosaceae (191), Burseraceae (175), Annonaceae (172) e Arecaceae (137) foram as mais representativas. No que tange às categorias de altura, os dados mostraram maior número de indivíduos, diversidade e grau de similaridade florística entre as classes 1 e 2. Licania caudata Prance, Duguetia flagellaris Huber, Monotagma tuberosum Hagberg, Protium apiculatum Swart e Pariana cf. campestris Aubl. foram as espécies de maior participação nos parâmetros verticais da regeneração natural e posição sociológica. O padrão agregado de distribuição espacial foi o predominante para as 30 espécies de maior participação na estrutura vertical da fitocenose.
\end{abstract}

PALAVRAS-CHAVE

Fitossociologia, Diversidade, Sub-bosque, Amazônia Central

\section{Floristic, phytosociological and ecological aspects of terra firme understory in central Amazonia, Amazonas state, Brazil.}

\begin{abstract}
Amazonian ecosystems generally have high species diversity. Afloristic and phytosociological analysis was undertaken in 0.05 ha of terra firme primary forest understory in Central Amazonia $\left(60^{\circ} 12^{\prime} 40^{\prime \prime} \mathrm{W}, 2^{\circ} 35^{\prime} 45^{\prime \prime} \mathrm{S}\right)$. Vascular plants were divided into four size categories: C1 (height $\leqslant 0.5 \mathrm{~m}), C 2(0.5 \mathrm{~m}<$ height $\leqslant 1.5 \mathrm{~m}), C 3(1.5 \mathrm{~m}<$ height $\leqslant 3.0 \mathrm{~m})$, and C4 (height $>3.0 \mathrm{~m}$, and CBH $>0,3 \mathrm{~m})$. The plot contained 2434 plants belonging to 67 families, 163 genera and 355 species. Families richest in species were Fabaceae (27), Mimosaceae (22), Lauraceae (21), Caesalpiniaceae (18) and Rubiaceae (18). The greatest density of individuals was found in the families Marantaceae (209), Chrysobalanaceae (198), Mimosaceae (191), Burseraceae (175), Annonaceae (172) and Arecaceae (137). The two smallest height categories (C1 and C2) had the most individuals, most species and highest floristic similarity between pairs of categories. Species showing highest ecological importance (based on natural regeneration index and sociological position value) in the understory were Licania caudata Prance, Duguetia flagellaris Huber, Monotagma tuberosum Hagberg, Protium apiculatum Swart and Pariana cf. campestris Aubl. An aggregated spatial distribution pattern was found for all but one of the thirty species with highest ecological importance among all size classes.
\end{abstract}

\section{KEYWORDS}

Phytosociology, Diversity, Understory, Central Amazonia

\footnotetext{
${ }^{1}$ Estudo executado com auxílio financeiro do CNPq, PPD-G7/LBA.

${ }^{2}$ Doutorando em Biotecnologia da Universidade Federal do Amazonas - UFAM. Manaus, AM. Email: arlem@inpa.gov.br

${ }^{3}$ Instituto Nacional de Pesquisas da Amazônia - INPA/CPBO. Manaus, AM. Email: iamaral@inpa.gov.br
} 


\section{ACTA \\ AMAZONICA}

ASPECTOS FLORÍSTICOS, FITOSSOCIOLÓGICOS E ECOLÓGICOS DE UM SUB-BOSQUE DE TERRA FIRME NA AMAZÔNIA CENTRAL, AMAZONAS, BRASIL.

\section{INTRODUÇÃO}

A regeneração natural define-se como sendo todos os descendentes de plantas arbóreas que se encontram entre $0,10 \mathrm{~m}$ de altura até o limite de diâmetro à altura do peito (DAP), estabelecido no levantamento estrutural (Finol, 1971; Rollet, 1978). O processo evolutivo da vegetação até a formação de uma floresta semelhante à primária, após o desmatamento parcial ou total de uma área, recebe o nome de regeneração natural, sendo que este processo pode durar até um século (Poggiani, 1989).

A análise da regeneração natural permite que sejam feitas inferências sobre a origem da floresta e previsões sobre seu desenvolvimento e aproveitamento, sob diferentes formas de tratamento (Carvalho, 1987). Por outro lado, o subbosque envolve a vegetação arbustiva e subarbustiva dos ambientes florestais. Essa vegetação forma um nicho ecológico de vital importância para o estabelecimento e desenvolvimento das espécies que irão constituir os demais estratos da floresta. Além disso, o sub-bosque representa 21 a $47 \%$ do total de espécies nas florestais tropicais (Gentry \& Dodson, 1987); apesar disso, na maioria das vezes, os estudos em comunidades florestais avaliam apenas a composição estrutural do componente arbóreo, relegando o estrato herbáceo-arbustivo ao esquecimento ou ao segundo plano.

Um dos poucos estudos voltado para a regeneração natural/sub-bosque em florestas de terra firme na região é o de Lima Filho et al. (2002), que destacou a ocorrência de Protium subserratum Engl. (30,6\%), Inga receptabilis (Vahl.) Wild. (15,9\%), Oenocarpus bacaba Mart. (12,4\%) e O. bataua Mart. (11,4\%). No mesmo estudo, os autores mencionam as famílias Selaginellaceae $(61,2 \%)$, Arecaceae $(4,04 \%)$, Burseraceae (3,4\%), Poaceae (3,2\%) e Mimosaceae $(2,2 \%)$ como sendo as mais abundantes. Diante da importância e da escassez de informações que envolvem tanto a regeneração natural como o estrato herbáceo-arbustivo nos ambientes florestais da região, o presente estudo visou avaliar a composição e diversidade florística do sub-bosque de uma comunidade florestal de platô na Amazônia Central, Amazonas, Brasil.

\section{MATERIAL E MÉTODOS}

\section{Área de estudo}

Trata-se de um ecossistema florestal de terra firme com área aproximada de $600 \mathrm{~km}^{2}$, localizada na Estação Experimental ZF-2, do Instituto Nacional de Pesquisas da Amazônia, INPA, situado a noroeste da cidade de Manaus, AM, tendo como limites a Rodovia BR-174
(Manaus - Boa Vista) e a bacia do Rio Cuieiras ( $2^{\circ} 35^{\prime} 45^{\prime \prime} \mathrm{S}$ e $\left.60^{\circ} 12^{\prime} 40^{\prime \prime} \mathrm{W}\right)$. A altitude média da área é de $45 \mathrm{~m}$ e o solo (Chauvel et al., 1987; Ferraz et al., 1998) do tipo Latossolo Amarelo Álico, de textura muito argilosa, caracterizado por um horizonte B poroso e fortemente micro-agregado. O clima, segundo a classificação de Köppen, é $A m W$ com temperatura média anual de $26,6^{\circ} \mathrm{C}$, umidade relativa de 75 a $86 \%$ e precipitação anual de 1.750 a $2.500 \mathrm{~mm}$ (Ribeiro \& Adis, 1984); os meses mais chuvosos vão de dezembro a maio, e os mais secos, de agosto a novembro.

\section{Desenho experimental, coleta e identificação do material botânico}

Foram demarcadas 20 sub-parcelas de $25 \mathrm{~m}^{2}$, alocadas cada uma no interior de dois transectos de $5.000 \mathrm{~m}^{2}$, distantes entre si $200 \mathrm{~m}$ (Figura 1), a pouco utilizados para estudos florísticos do componente arbóreo (Oliveira et al., dados não publicados). A abordagem dos espécimes arbóreos, arbustivos, herbáceos, palmeiras e lianas seguiu o critério de classificação em categorias de altura (Higuchi, 1985; Lima-Filho, 1995; Lima-Filho et al., 2002), adotandose as seguintes classes: $\mathrm{C} 1$ (altura $\leqslant 0,5 \mathrm{~m}), \mathrm{C} 2(0,5<$ altura $\leqslant 1,5 \mathrm{~m}), \mathrm{C} 3(1,5<$ altura $\leqslant 3,0 \mathrm{~m})$ e C 4 (altura $>3 \mathrm{~m}$ e CAP $<0,3 \mathrm{~m}$ ).

Depois de secas por 48 horas em estufa $\left(70^{\circ} \mathrm{C}\right)$, as amostras botânicas foram identificadas a partir de referência especializada (Ribeiro et al., 1999) e/ou por comparação com as exsicatas disponíveis no herbário do INPA. As amostras não identificadas com nomes científicos receberam códigos de morfotipo. Após o processo de identificação taxonômica, o material será devidamente guardado por um período de até dois anos após a publicação dos resultados.

Os nomes botânicos apresentados nesse estudo foram conferidos com a página da WEB do Missouri Botanical Garden (http://mobot.mobot.org/W3T/Search/vast.html).
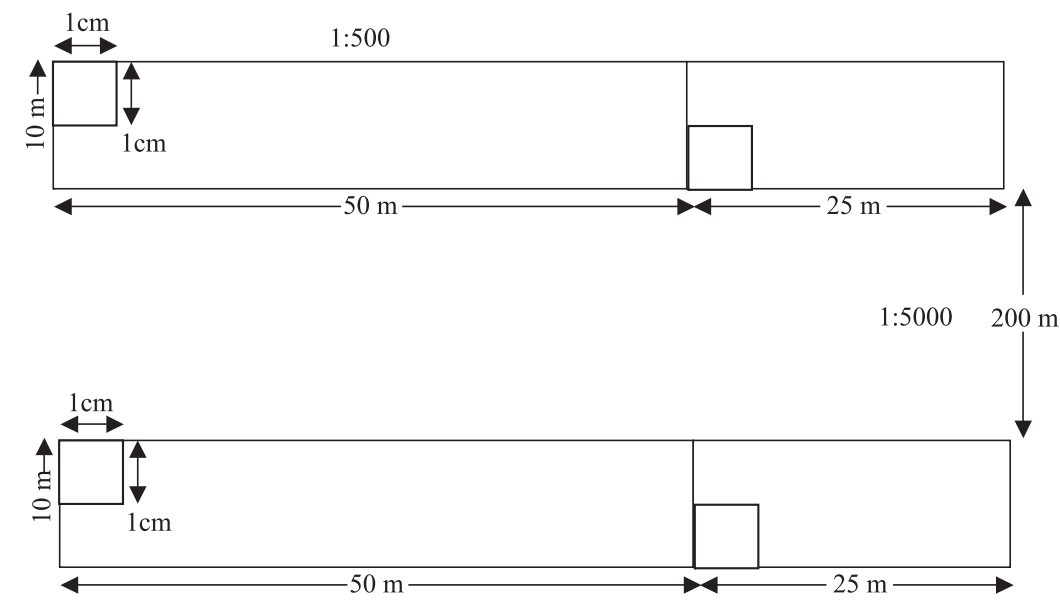

Figura 1 - Croqui resumido dos transectos de $500 \times 10 \mathrm{~m}$ mostrando a demarcação das sub-parcelas de $5 \times 5 \mathrm{~m}$ no interior de 20 parcelas de $50 \times 10 \mathrm{~m}$. 


\section{Parâmetros fitossociológicos}

a) Estrutura horizontal (Lamprecht, 1964)

Densidade Absoluta

$\mathrm{DA}_{\mathrm{i}}=\mathrm{N}_{\mathrm{i}}$

Densidade Relativa

$$
\mathrm{DR}_{\mathrm{i}}=\left(\mathrm{DA}_{\mathrm{i}} / \sum_{\mathrm{i}=1}^{\mathrm{S}} \mathrm{N}_{\mathrm{i}}\right) \times 100
$$

Freqüência Absoluta

$\mathrm{FA}_{\mathrm{i}}=\left(\mathrm{NP}_{\mathrm{i}} / \mathrm{NP}_{\mathrm{t}}\right) \times 100$

Freqüência Relativa

$$
\mathrm{DR}_{\mathrm{i}}=\left(\mathrm{FA}_{\mathrm{i}} / \sum_{\mathrm{i}=1}^{\mathrm{S}} \mathrm{FA}\right) \times 100
$$

Onde:

$\mathrm{N}_{\mathrm{i}}=$ Número de indivíduos amostrados da i-ésima espécie por ha;

$\sum_{\mathrm{i}=1}^{\mathrm{S}} \mathrm{N}_{\mathrm{i}}=$ Número total de indivíduos amostrados por ha;

$\sum_{\mathrm{i}=1}^{\mathrm{S}} \mathrm{FA}_{1}=$ Soma das freqüências absolutas das espécies amostras;

$\mathrm{NP}_{\mathrm{i}}=$ Número de parcelas em que ocorreu a i-ésima espécie; $\mathrm{P}_{\mathrm{t}}=$ Número total de parcelas.

Estrutura vertical: Posição sociológica e Regeneração natural/Sub-bosque (Finol, 1971)

Posição Sociológica Absoluta

$\left.\left.\mathrm{PSA}_{\mathrm{i}}=\left(\mathrm{VF}_{(\mathrm{C} 1)} \times \mathrm{N}_{\mathrm{i}(\mathrm{C} 1)}\right)+\mathrm{VF}_{(\mathrm{C} 2)} \times \mathrm{N}_{\mathrm{i}(\mathrm{C} 2)}\right)+\mathrm{VF}_{(\mathrm{C} 3)} \times \mathrm{N}_{\mathrm{i}(\mathrm{C} 3)}\right)$

$\left.+\mathrm{VF}_{(\mathrm{C} 4)} \times \mathrm{N}_{\mathrm{i}(\mathrm{C} 4)}\right)$, em que:

$\mathrm{VF}=\mathrm{NC} / \sum_{\mathrm{i}=1} \mathrm{~N}_{\mathrm{i}}$

Posição Sociológica Relativa

$\mathrm{PSR}_{\mathrm{i}}=\left(\mathrm{PSA}_{\mathrm{i}} / \sum_{\mathrm{i}=1}^{\mathrm{S}} \mathrm{PSA}_{\mathrm{i}}\right) \times 100$

Classe absoluta de altura da regeneração natural/sub-bosque

$\operatorname{CAARN}_{i}=\sum_{i=1}^{j}\left(n_{i j} \times N_{j}\right) / N T$
Classe relativa de altura da regeneração natural/sub-bosque

CRARN $_{i}=\left(\right.$ CATRN $_{i} / \sum_{i=1}^{K}$ CAARN $\left._{i}\right) \times 100$

Regeneração natural/sub-bosque relativa

$\mathrm{RNR}_{\mathrm{i}}=\left(\mathrm{DRRN}_{\mathrm{i}}+\mathrm{FRRN}_{\mathrm{i}}+\mathrm{CRARN}_{\mathrm{i}}\right) / 4$

Onde:

$\mathrm{VF}=$ Valor fitossociológico do i-ésima classe de tamanho;

$\mathrm{NC}=$ Número de indivíduos amostrados na i-ésima classe de altura;

$\mathrm{C}_{1}, \mathrm{C}_{2}, \mathrm{C}_{3}, \mathrm{C}_{4}=$ Classes de tamanhos $1,2,3$ e 4 , respectivamente;

$\sum_{i=1}^{S} \mathrm{PSA}_{\mathrm{i}}=$ Soma das posições sociológicas absolutas;

$\mathrm{j}=$ Número de classes de altura;

$\mathrm{n}_{\mathrm{ij}}=$ Número de indivíduos da i-ésima espécie na j-ésima classe de altura;

$\mathrm{N}_{\mathrm{j}}$ = Número de indivíduos na j-ésima classe de altura;

$\mathrm{K}$ = número de espécies;

$\mathrm{DRRN}_{\mathrm{i}}=$ densidade relativa da regeneração natural/subbosque para a i-ésima espécie;

$\mathrm{FRRN}_{\mathrm{i}}=$ freqüência relativa da regeneração natural/subbosque para a i-ésima espécie.

\section{Padrão de distribuição espacial}

Para determinar o padrão de distribuição das populações de cada espécie, adotou-se o Índice de Morisita padronizado (Ip), expresso pelas seguintes fórmulas (Krebs, 1989):

$$
\begin{aligned}
& \text { Ip }=0,5+0,5(\mathrm{Id}-\mathrm{Mc} / \mathrm{n}-\mathrm{Mc}), \text { quando } \mathrm{Id} \geqslant \mathrm{Ma}>1,0 ; \\
& \mathrm{Ip}=0,5(\mathrm{Id}-1 / \mathrm{Mu}-1), \text { quando } \mathrm{Ma}>\mathrm{Id} \geqslant 1,0 ; \\
& \mathrm{Ip}=0,5(\mathrm{Id}-1 / \mathrm{Mu}-1), \text { quando } 1,0>\mathrm{Id}>\mathrm{Mu} ; \\
& \mathrm{Ip}=-0,5+0,5(\mathrm{Id}-\mathrm{Mu} / \mathrm{Mu}), \text { quando } 1,0>\mathrm{Mu}>\mathrm{Id} .
\end{aligned}
$$

Onde: Id = Índice de dispersão de Morisita (Id $=\mathrm{n}\left[\sum\right.$ $\left.\left.\mathbf{x}_{\mathrm{i}}^{2}-\sum \mathrm{x}_{\mathrm{i}} /\left(\sum \mathrm{x}_{\mathrm{i}}\right)^{2}-\sum \mathrm{x}_{\mathrm{i}}\right]\right) ; \mathrm{n}=$ Número de parcelas; $\sum \mathrm{x}_{\mathrm{i}}=$ Somatória do número de indivíduos da espécie i nas 20 parcelas $=\mathrm{x}_{1}+\mathrm{x}_{2}+\mathrm{x}_{3} \ldots+\mathrm{x}_{20} ; \sum \mathrm{x}_{\mathrm{i}}^{2}=$ Somatória do número de indivíduos da espécie $\mathrm{i}$ ao quadrado nas 20 parcelas; $\mathrm{Mu}=$ Índice de uniformidade de Morisita $(\mathrm{Mu}=$ $\left.\chi_{0,975}^{2}-\mathrm{n}+\sum \mathrm{x}_{\mathrm{i}} /\left(\sum \mathrm{x}_{\mathrm{i}}\right)-1\right) ; \mathrm{Ma}=$ Índice de agregação de Morisita $\left(\mathrm{Ma}=\chi_{0,025}^{2}-\mathrm{n}+\sum \mathrm{x}_{\mathrm{i}} /\left(\sum \mathrm{x}_{\mathrm{i}}\right)-1\right) ; \chi_{0,975}^{2} \mathrm{e} \chi_{0,025}^{2}$ $=$ Valores do Qui-quadrado tabelados com n-1 graus de liberdade que têm 97,5\% e 2,5\% da área para a direita. Valores de Ip $\geqslant 0,5$ indicam distribuição espacial agregada; I $p \leqslant-0,5$ designam distribuição uniforme; Ip entre $+0,5$ e - 0,5 indicam padrão aleatório (Krebs, 1989). 


\section{ACTA AMAZONICA}

ASPECTOS FLORÍSTICOS, FITOSSOCIOLÓGICOS E ECOLÓGICOS DE UM SUB-BOSQUE DE TERRA FIRME NA AMAZÔNIA CENTRAL, AMAZONAS, BRASIL.

\section{Diversidade florística}

As diversidades foram estimadas de acordo com os Índices de Riqueza de Espécie e de Shannon-Wiener (Magurran, 1988). O primeiro Índice foi obtido da razão entre o número de espécies acumuladas e o tamanho da área amostrada $\left(\mathrm{m}^{2}\right)$, enquanto o segundo, pela fórmula: $H^{\prime}=-\sum\left(p_{i}\right)\left(\log _{e} p_{i}\right)$, onde $\mathrm{p}_{\mathrm{i}}=(\mathrm{ni} / \mathrm{N})$ é a probabilidade de que um indivíduo amostrado pertença a espécie $i ; n_{i}=n^{\circ}$ total de indivíduos da espécie i; $N$ $=\mathrm{n}^{\circ}$ total de indivíduos amostrados na área; a equitabilidade (uniformidade) foi obtida segundo a fórmula E' $=\mathrm{H}^{\prime} / \log _{\mathrm{e}} \sum$ (Magurran, 1988), onde $\sum=\mathrm{n}^{\circ}$ de espécies.

\section{Similaridade florística}

As similaridades entre as classes de altura foram comparadas por intermédio do índice qualitativo de Sorensen (MüellerDombois \& Ellemberg, 1974).

\section{RESULTADOS E DISCUSSÃO}

\section{Aspectos fisionômicos}

A vegetação da Estação Experimental ZF-2 é bastante heterogênea, com predomínio das famílias Lecythidaceae, Sapotaceae, Fabaceae, Euphorbiaceae, Chrysobalanaceae, Mimosaceae, Moraceae, Annonaceae, Burseraceae e
Caesalpiniaceae (Jardim \& Hosokawa, 1986/87; Rankin-de-Mérona et al., 1992; Higuchi et al., 1998); as espécies mais importantes (Oliveira et al., dados não publicados) são: Eschweilera micrantha (O.Berg.) Miers (matá-matá), Pouteria gongrijpii Eyma (abiu), Chrysophyllum sanguinolentum (Pierre) Baehni (ucuquirana), Protium apiculatum Swart (breu-vermelho), Eschweilera atropetiolata S.A. Mori (castanha-vermelha), Swartzia reticulata Ducke (arabá-preto), Licania davillifolia Benoist (caraipé), Eschweilera coriacea (DC.) S.A. Mori (matá-matá), Aspidosperma oblongum A. DC (carapanaúba) e Dinizia excelsa Ducke (angelimpedra). Ainda, segundo os autores, mais de 50\% das árvores encontram-se entre 14 e $25 \mathrm{~m}$, sendo que Abarema mataybifolia (Sandw.) Barneby \& Grimes (tento), Leonia glycycarpa Ruiz \& Pav. (leonia), Swartzia reticulata Ducke (arabá-preto) e Aspidosperma oblongum A. DC (carapanaúba) são as únicas espécies a possuírem valores superiores a $90 \mathrm{~cm}$ de DAP.

\section{Composição florística da regeneração natural e sub-bosque}

Nos $500 \mathrm{~m}^{2}$ de vegetação amostrados, encontrou-se 2.434 indivíduos pertencentes a 67 famílias, 164 gêneros e 356 espécies, das quais três ficaram indeterminadas (Tabela 1). Comparando esses resultados com outros obtidos na região (Vieira, 1989; Lima Filho, 1995; Lima Filho et al., 2002), notouse que a área é bem mais diversificada em termos de famílias e espécies, apesar do local amostrado possuir dimensões inferiores em relação às outras áreas avaliadas.

Tabela 1 - Listagem das espécies vegetais presentes no sub-bosque de uma floresta de terra firme na Amazônia Central, Amazonas, Brasil. Onde: $\mathrm{C} 1=$ espécies amostradas na classe 1 (altura $\leqslant 0,5 \mathrm{~m}), \mathrm{C} 2=$ espécies amostradas na classe $2(0,5<$ altura $\leqslant 1,5 \mathrm{~m})$, C3 = espécies amostradas na classe $3(1,5<$ altura $\leqslant 3,0 \mathrm{~m}), \mathrm{C} 4=$ espécies amostradas na classe 4 (altura $>3 \mathrm{~m}$ e CAP $<0,3 \mathrm{~m})$, * = espécies "localmente raras".

\begin{tabular}{|c|c|c|c|c|c|c|}
\hline Famílias & Nomes Científicos & Hábitos & C1 & $\mathrm{C} 2$ & C3 & C4 \\
\hline Anacardiaceae & Anacardium cf. spruceanum Benth. ex Engl.* & árvore & 1 & - & - & - \\
\hline Annonaceae & Bocageopsis multiflora (Mart.) R.E. Fr. & $"$ & 2 & - & - & 1 \\
\hline$"$ & Duguetia flagellaris Huber & árvore & 47 & 46 & 20 & 13 \\
\hline$"$ & D. ulei (Diels) R.E. Fr.* & $"$ & 1 & - & - & - \\
\hline " & Fusaea longifolia (Aubl.) Saff. & árvore & - & - & 2 & - \\
\hline$"$ & Guatteria duckeana R.E. Fr. & $"$ & - & 5 & - & - \\
\hline$"$ & G. foliosa Benth. & $"$ & - & 1 & 4 & - \\
\hline$"$ & G. insculpta R.E. Fr. & $"$ & 9 & - & - & - \\
\hline$"$ & Guatteria sp. & $"$ & - & 2 & - & 2 \\
\hline$"$ & Guatteriopsis blepharophylla (Mart.) R.E. Fr. & árvore & 5 & - & 1 & 1 \\
\hline$"$ & Oxandra xylopioides Diels. & $"$ & 6 & 1 & - & 2 \\
\hline Apocynaceae & Ambelania sp.* & árvore & - & 1 & - & - \\
\hline$"$ & Aspidosperma discolor A. DC. & árvore & 4 & 1 & - & - \\
\hline$"$ & Geissospermum sericeum Benth. \& Hook. ex Miers & $"$ & - & 1 & - & 1 \\
\hline " & G. urceolatum A.H. Gentry* & $"$ & - & 1 & - & - \\
\hline$"$ & Lacmellea sp. & $"$ & 3 & - & - & - \\
\hline " & Mandevilla scabra (Roem. \& Schult.) K. Schum.* & liana & 1 & - & - & - \\
\hline$"$ & Mandevilla sp.* & $"$ & - & - & - & 1 \\
\hline Araceae & Heteropsis sp.* & hemiepífita & 1 & - & - & - \\
\hline
\end{tabular}




\section{ACTA \\ AMAZONICA}

ASPECTOS FLORÍSTICOS, FITOSSOCIOLÓGICOS E ECOLÓGICOS DE UM

SUB-BOSQUE DE TERRA FIRME NA AMAZÔNIA CENTRAL, AMAZONAS, BRASIL.

\begin{tabular}{|c|c|c|c|c|c|c|}
\hline Famílias & Nomes Científicos & Hábitos & C1 & $\mathrm{C} 2$ & $\mathrm{C} 3$ & $\mathrm{C} 4$ \\
\hline$"$ & Philodendron elaphoglossoides Schott* & $"$ & - & - & 1 & - \\
\hline Araliaceae & Schefflera morototoni (Aubl.) Frodin* & árvore & 1 & - & - & - \\
\hline Arecaceae & Astrocaryum gynacanthum Mart. & palmeira & 7 & 5 & 5 & 4 \\
\hline$"$ & A. sciophilum (Miq.) Pulle & $"$ & 10 & 7 & 3 & - \\
\hline$"$ & Attalea attaleoides (Barb. Rodr.) Wess. Boer & $"$ & 1 & - & 2 & 2 \\
\hline$"$ & Attalea sp. & $"$ & 7 & 1 & 4 & - \\
\hline$"$ & Bactris maraja Mart. & $"$ & - & 2 & - & - \\
\hline$"$ & Bactris sp. & $"$ & 3 & 1 & 1 & - \\
\hline$"$ & Geonoma deversa (Poit.) Kunth & $"$ & 4 & - & 3 & - \\
\hline$"$ & Geonoma sp. & $"$ & 4 & - & 4 & - \\
\hline$"$ & Oenocarpus bacaba Mart. & $"$ & 33 & 1 & 1 & 2 \\
\hline " & O. minor Mart. & $"$ & 6 & 4 & - & - \\
\hline$"$ & Syagrus inajai (Spruce) Becc. & $"$ & 5 & 3 & 1 & 1 \\
\hline Bignoniaceae & Arrabidaea nigrescens Sandwith. & liana & - & 2 & - & - \\
\hline$"$ & A. bilabiata (Sprague) Sandwith & $"$ & 1 & 1 & - & - \\
\hline$"$ & A. egensis Bureau \& $\mathrm{K}$. Schum. & $"$ & 15 & 11 & - & 2 \\
\hline$"$ & A. fanshawei Sandw. & $"$ & - & 2 & 1 & - \\
\hline$"$ & Memora adenophora Sandw. & $"$ & 8 & 4 & 1 & 1 \\
\hline " & M. cf. magnifica (Mart. ex DC.) Bureau & $"$ & 6 & 7 & 1 & 2 \\
\hline " & M. flaviflora (Miq.) Pulle* & $"$ & 1 & - & - & - \\
\hline$"$ & M. longilinea A.Sampaio & $"$ & 8 & 5 & - & 1 \\
\hline$"$ & M. moringifolia Sandw. & $"$ & 2 & - & - & - \\
\hline$"$ & Memora sp.* & $"$ & - & - & 1 & - \\
\hline \multirow[t]{2}{*}{ Bombacaceae } & Quararibea ochrocalyx (K. Schum.) Vischer* & árvore & - & - & - & 1 \\
\hline & Scleronema micranthum Ducke & $"$ & 3 & - & - & - \\
\hline \multirow[t]{2}{*}{ Boraginaceae } & Cordia cf. sprucei Mez & $"$ & 1 & - & - & - \\
\hline & C. nodosa Lam. & arbusto & 1 & 1 & 1 & - \\
\hline Burseraceae & Protium apiculatum Swart & árvore & 70 & 16 & 1 & 6 \\
\hline$"$ & P. aracouchini (Aubl.) March. & $"$ & 6 & 1 & - & - \\
\hline$"$ & P. cf. carolense Daly* & $"$ & 1 & - & - & - \\
\hline$"$ & P. cf. grandifolium Engl. & $"$ & 6 & - & - & 2 \\
\hline$"$ & P. divaricatum Engl. & $"$ & 18 & 5 & 2 & 3 \\
\hline$"$ & P. hebetatum Daly & $"$ & 16 & 7 & - & 4 \\
\hline " & P. hostmannii (Miq.) Engl. & $"$ & 3 & - & - & 1 \\
\hline " & P. tenuifolium (Engl.) Engl. & $"$ & 6 & - & - & - \\
\hline$"$ & P. trifoliolatum Engl. & árvore & - & 1 & 1 & 1 \\
\hline Caesalpiniaceae & Bauhinia alata Ducke* & liana & 1 & - & - & - \\
\hline$"$ & B. cf. guianensis Aubl. & $"$ & 2 & - & - & - \\
\hline$"$ & B. cf. splendens Kunth & $"$ & 3 & 4 & - & 1 \\
\hline$"$ & B. platycalyx Benth. & $"$ & 5 & - & - & - \\
\hline$"$ & Dialium guianense (Aubl.) Sandw. * & árvore & 1 & - & - & - \\
\hline$"$ & Dimorphandra caudata Ducke* & $"$ & - & - & 1 & - \\
\hline$"$ & Elizabetha sp. & árvore & 1 & 1 & - & - \\
\hline$"$ & Indeterminada & - & - & - & 2 & - \\
\hline$"$ & Macrolobium gracile Spruce ex Benth. & árvore & 12 & - & - & 1 \\
\hline$"$ & M. limbatum Spruce ex Benth. * & " & 1 & - & - & - \\
\hline$"$ & M. microcalix Ducke* & $"$ & 1 & - & - & - \\
\hline$"$ & M. prancei R.S. Cowan* & $"$ & - & - & - & 1 \\
\hline$"$ & Macrolobium sp. * & $"$ & - & - & - & 1 \\
\hline
\end{tabular}




\begin{tabular}{|c|c|c|c|c|c|c|}
\hline Famílias & Nomes Científicos & Hábitos & C1 & $\mathrm{C} 2$ & C3 & C4 \\
\hline$"$ & Peltogyne paniculata Benth. & $"$ & 17 & - & 1 & - \\
\hline$"$ & Sclerolobium guianense Benth. & $"$ & 3 & - & - & - \\
\hline$"$ & Sclerolobium sp. 1 & $"$ & 35 & 4 & - & 1 \\
\hline$"$ & Sclerolobium sp. $2^{*}$ & $"$ & 1 & - & - & - \\
\hline$"$ & Tachigali sp. 1 & $"$ & 5 & 3 & 1 & 1 \\
\hline$"$ & Tachigali sp. 2 & $"$ & - & 1 & - & 2 \\
\hline Cecropiaceae & Pourouma bicolor Mart. & árvore & 8 & - & - & - \\
\hline$"$ & P. ferruginea Standl. & $"$ & - & - & - & 2 \\
\hline$"$ & P. guianensis Aubl. & $"$ & 9 & - & 1 & 1 \\
\hline$"$ & P. tomentosa Miq. * & $"$ & - & 1 & - & - \\
\hline$"$ & Pourouma sp. * & $"$ & - & - & 1 & - \\
\hline Chrysobalanaceae & Couepia abovata Ducke* & $"$ & - & 1 & - & - \\
\hline$"$ & C. magnoliifolia Benth. ex Hook. f. & $"$ & 5 & - & - & - \\
\hline$"$ & Hirtella araguaiensis Prance & $"$ & - & 5 & 2 & 1 \\
\hline$"$ & H. duckei Huber & $"$ & 5 & 1 & 1 & 3 \\
\hline$"$ & H. racemosa Lam. & $"$ & 2 & - & - & 1 \\
\hline$"$ & Licania canescens Benoist & $"$ & 7 & 2 & 1 & - \\
\hline$"$ & L. caudata Prance & $"$ & 142 & 3 & - & - \\
\hline " & L. cf. cidii Prance & $"$ & - & 2 & - & - \\
\hline$"$ & L. davillifolia Benoist*. & $"$ & - & - & - & 1 \\
\hline " & L. densiflora Kleinhoonte & $"$ & 3 & 2 & 1 & 2 \\
\hline$"$ & Licania sp. & $"$ & 3 & - & 1 & 1 \\
\hline Clusiaceae & Tovomita pyrifolia Planch. \& Triana* & $"$ & 1 & - & - & - \\
\hline Combretaceae & Combretum laxum Aubl. & liana & - & - & - & 2 \\
\hline Connaraceae & Connarus cf. punctatus Planch. * & $"$ & - & 1 & - & - \\
\hline$"$ & C. erianthus Benth. ex Baker & $"$ & 12 & 5 & - & 1 \\
\hline " & C. perrottetii (DC.) Planch. * & $"$ & 1 & - & - & - \\
\hline$"$ & Connarus sp. 1 & $"$ & - & 3 & - & - \\
\hline " & Connarus sp. 2 & $"$ & 8 & 1 & 1 & 1 \\
\hline$"$ & Pseudoconnarus cf. macrophyllus (Poepp. \& Endl.) Radlk. & liana & 5 & - & - & 2 \\
\hline " & Rourea cuspidata Benth. ex Backer & liana & 5 & 2 & - & 1 \\
\hline " & P. rhynchosioides (Poepp. \& Endl.) Radlk. * & $"$ & - & 1 & - & - \\
\hline$"$ & Pseudoconnarus sp. & $"$ & - & - & 1 & 1 \\
\hline " & Rourea cuspidata Benth. ex Baker & $"$ & 2 & 1 & - & - \\
\hline Convolvulaceae & Dicranostyles laxa Ducke* & $"$ & - & 1 & - & - \\
\hline$"$ & D. holostyla Ducke* & $"$ & - & 1 & - & - \\
\hline$"$ & Indeterminada* & $"$ & - & 1 & - & - \\
\hline$"$ & Maripa sp. * & $"$ & - & - & 1 & - \\
\hline Cyperaceae & Mapania sylvatica Aubl. & erva & 7 & - & - & - \\
\hline Dennstaedtiaceae & Lindsaea lancea (L.) Bedd. & $"$ & 32 & - & - & - \\
\hline Dichapetalaceae & Dichapetalum rugosum (Vahl) Prance* & liana & 1 & - & - & - \\
\hline " & Tapura guianensis Aubl. & árvore & 3 & 4 & - & 2 \\
\hline Dilleniaceae & Davilla kunthii St. - Hil. & liana & 1 & - & - & 1 \\
\hline$"$ & Doliocarpus amazonicus Sleumer* & $"$ & 1 & - & - & - \\
\hline$"$ & D. brevipedicellatus Garcke & $"$ & 4 & - & - & - \\
\hline$"$ & D. dentatus (Aubl.) Standl. & $"$ & 12 & 2 & - & 1 \\
\hline$"$ & D. magnificus Sleumer & $"$ & 7 & 1 & - & - \\
\hline$"$ & D. novogranatensis Kubitzki* & $"$ & - & - & - & 1 \\
\hline Elaeocarpaceae & Sloanea excelsa Ducke* & árvore & - & - & - & 1 \\
\hline
\end{tabular}




\begin{tabular}{|c|c|c|c|c|c|c|}
\hline Famílias & Nomes Científicos & Hábitos & C1 & $\mathrm{C2}$ & $\mathrm{C3}$ & C4 \\
\hline$"$ & S. schomburgkii Spruce ex Benth. & $"$ & 6 & - & - & 1 \\
\hline \multirow{2}{*}{$\begin{array}{l}\text { Erythroxylaceae } \\
\text { " }\end{array}$} & Erythroxylum amplum Benth. & erva & 4 & - & - & - \\
\hline & E. citrifolium St.- Hil. * & árvore & - & 1 & - & - \\
\hline Euphorbiaceae & Conceveiba guianensis Aubl. & $"$ & 11 & - & - & - \\
\hline " & C. hostmannii Benth. & " & 12 & - & - & - \\
\hline " & Croton cuneatus Klotz. & erva & 2 & - & - & - \\
\hline " & C. matourensis Aubl. * & $"$ & - & 1 & - & - \\
\hline " & Mabea angularis G. den Hollander & árvore & 5 & 11 & 2 & 3 \\
\hline$"$ & M. cf. nitida Spruce ex Benth. * & $"$ & 1 & - & - & - \\
\hline$"$ & M. occidentalis Benth. * & $"$ & 1 & - & - & - \\
\hline$"$ & M. speciosa Müll. Arg. & $"$ & - & - & 1 & 2 \\
\hline " & Micrandra rossiana R.E. Schult. * & $"$ & 1 & - & - & - \\
\hline$"$ & Pogonophora schomburgkiana Miers ex Benth. & $"$ & - & - & 1 & - \\
\hline " & Nealchornea yapurensis Huber & " & 2 & - & - & - \\
\hline " & Sandwithia guianensis Lanj. * & " & - & - & 1 & - \\
\hline " & Senefeldera macrophylla Ducke & $"$ & - & - & - & 2 \\
\hline Fabaceae & Andira trifoliolata Ducke* & $"$ & - & - & - & 1 \\
\hline$"$ & Bocoa alterna (Benth.) R.S. Cowan & árvore & 10 & 3 & - & - \\
\hline$"$ & B. mollis (Benth.) R.S. Cowan & $"$ & 2 & - & - & - \\
\hline$"$ & B. viridiflora (Ducke) R.S. Cowan & $"$ & 1 & 1 & 1 & 2 \\
\hline " & Clitoria cf. grandifolia Ducke* & liana & - & - & - & 1 \\
\hline " & C. leptostachya Benth. & $"$ & 1 & 2 & - & 1 \\
\hline$"$ & Dalbergia sp. * & $"$ & 1 & - & - & - \\
\hline$"$ & Deguelia sp. 1 & $"$ & 1 & 1 & - & - \\
\hline " & Deguelia sp. 2 & $"$ & 1 & 1 & - & 1 \\
\hline " & Deguelia sp. $3^{*}$ & " & - & - & 1 & - \\
\hline " & Derris floribunda (Benth.) Ducke & " & - & 1 & 2 & 2 \\
\hline " & Derris sp. & $"$ & - & 4 & 2 & 1 \\
\hline " & Dipteryx odorata (Aubl.) Willd. * & árvore & - & - & 1 & - \\
\hline " & Machaerium amplum Benth. * & liana & 1 & - & - & - \\
\hline " & M. aristulatum (Spruce ex Benth.) Ducke* & $"$ & - & - & - & 1 \\
\hline$"$ & Machaerium sp. & $"$ & 3 & 1 & - & - \\
\hline " & Swartzia arborescens (Aubl.) Pittier & árvore & 2 & - & - & - \\
\hline$"$ & S. cf. corrugata Benth. & $"$ & 4 & 3 & - & 1 \\
\hline " & S. cf. ingifolia Ducke & $"$ & 3 & - & - & - \\
\hline " & S. cuspidata Spruce ex Benth. & $"$ & 3 & 1 & - & 1 \\
\hline " & S. longistipitata Ducke & $"$ & 1 & - & 1 & - \\
\hline " & S. macrocarpa Spruce ex Benth. & $"$ & 1 & - & 1 & 1 \\
\hline " & S. recurva Poepp. & $"$ & 3 & - & - & - \\
\hline " & S. reticulata Ducke & $"$ & 3 & 4 & 3 & 1 \\
\hline$"$ & S. schomburgkii Benth. & $"$ & 3 & 2 & - & - \\
\hline$"$ & Swartzia ulei Harms & $"$ & 1 & - & 2 & - \\
\hline " & Swartzia sp. & $"$ & 3 & - & - & - \\
\hline Flacourtiaceae & Carpotroche crispidentata Ducke & $"$ & 2 & 2 & - & - \\
\hline " & Casearia cf. negrensis Eichler* & árvore & 1 & - & - & - \\
\hline " & C. javitensis Kunth & " & - & 2 & - & 1 \\
\hline Heliconiaceae & Heliconia acuminata Rich. & erva & 22 & 6 & - & - \\
\hline Hippocrateaceae & Salacia impressifolia (Miers) A.C. Sm. * & liana & - & 1 & - & - \\
\hline & S. insignis A.C. Sm. & $"$ & 9 & 6 & - & - \\
\hline
\end{tabular}




\begin{tabular}{|c|c|c|c|c|c|c|}
\hline Famílias & Nomes Científicos & Hábitos & C1 & C2 & C3 & $\mathrm{C} 4$ \\
\hline$"$ & S. juruana Loes. & $"$ & 1 & 3 & - & 2 \\
\hline$"$ & Salacia sp. & $"$ & - & 4 & - & - \\
\hline " & Tontelea cylindrocarpa (A.C. Sm.) A.C. Sm. & liana & 3 & 1 & 1 & - \\
\hline Humiriaceae & Duckesia verrucosa (Ducke) Cuatrec. & árvore & - & 2 & 1 & 1 \\
\hline " & Endopleura uchi (Huber) Cuatrec. & $"$ & 1 & 1 & - & 1 \\
\hline " & Vantanea guianensis (Aubl.) Ducke & " & 1 & - & - & 1 \\
\hline " & V. macrocarpa Ducke* & $"$ & 1 & - & - & - \\
\hline Lacistemaceae & Lacistema sp. * & árvore & - & 1 & - & - \\
\hline Lauraceae & Aiouea maguireana (P. Allen) S.S. Renner* & $"$ & - & 1 & - & - \\
\hline$"$ & Aniba canelilla (Kunth) Mez. & $"$ & 1 & 1 & - & - \\
\hline " & A. megaphylla Mez. * & $"$ & 1 & - & - & - \\
\hline " & Aniba sp. * & $"$ & - & - & 1 & - \\
\hline " & Endlicheria bracteata Mez* & " & - & 1 & - & - \\
\hline " & E. sprucei (Meisn.) Mez & $"$ & 2 & - & - & - \\
\hline " & Licaria cf. rodriguesii Kurz & " & - & 1 & 1 & 2 \\
\hline " & L. chrysophylla (Meissn.) Kosterm.* & $"$ & - & 1 & - & - \\
\hline " & L. guianensis Aubl. & $"$ & 1 & - & 1 & 1 \\
\hline$"$ & Mezilaurus cf. synandra (Mez.) Kosterm. ${ }^{*}$ & $"$ & 1 & - & - & - \\
\hline " & M. duckei Van der Werff & " & 3 & - & - & - \\
\hline " & Ocotea aciphylla (Nees) Mez & $"$ & 1 & - & 1 & - \\
\hline " & O. amazonica (Meissn.) Mez & $"$ & 5 & 1 & - & - \\
\hline$"$ & O. argyrophylla Ducke* & $"$ & 1 & - & - & - \\
\hline " & O. caudata (Nees) Mez & $"$ & 3 & 1 & 1 & 1 \\
\hline$"$ & O. cf. cujumari Mart. * & $"$ & 1 & - & - & - \\
\hline " & O. douradensis Vattimo-Gil & $"$ & 1 & - & 2 & - \\
\hline$"$ & O. guianensis Aubl. & $"$ & 3 & 3 & 2 & - \\
\hline " & O. longifolia Kunth & $"$ & 22 & 2 & - & 1 \\
\hline " & Ocotea sp. 1 & $"$ & 4 & 1 & - & - \\
\hline " & Ocotea sp. 2 & $"$ & - & 4 & - & - \\
\hline Lecythidaceae & Eschweilera apiculata (Miers) A.C. Sm. & $"$ & 3 & 1 & - & - \\
\hline " & E. atropetiolata S.A.Mori* & $"$ & - & 1 & - & - \\
\hline$"$ & E. bracteosa (Poepp. ex O.Berg) Miers & $"$ & 24 & - & 3 & 1 \\
\hline " & E. coriacea (DC.) S.A. Mori & " & 14 & 3 & - & 2 \\
\hline " & E. decolorans Sandw. & $"$ & - & 3 & - & - \\
\hline$"$ & E. grandiflora (Aubl.) Sandw. * & $"$ & 1 & - & - & - \\
\hline$"$ & E. laevicarpa S.A. Mori & $"$ & - & 4 & 1 & - \\
\hline " & E. micrantha (O.Berg) Miers & $"$ & 8 & - & - & 2 \\
\hline$"$ & E. pedicellata (Rich.) S.A. Mori & $"$ & 4 & 3 & - & - \\
\hline$"$ & Eschweilera sp. & $"$ & 2 & - & - & 2 \\
\hline$"$ & Gustavia augusta L. & $"$ & 2 & 3 & 5 & - \\
\hline Loganiaceae & Strychnos jobertiana Baill. & liana & 2 & 2 & 1 & 1 \\
\hline Malpighiaceae & Heteropteris sp. * & arbusto & - & - & - & 1 \\
\hline Marantaceae & Calathea mansonis Körn. & erva & 22 & - & - & - \\
\hline$"$ & Calathea sp. & $"$ & 2 & - & - & - \\
\hline$"$ & Ischnosiphon arouma (Aubl.) Körn. & $"$ & 5 & 16 & - & - \\
\hline$"$ & I. cannoideus L. Andersson & $"$ & 17 & 12 & 3 & - \\
\hline " & I. gracilis (Rudge) Körn. & $"$ & 14 & - & - & - \\
\hline$"$ & Ischnosiphon sp. & $"$ & - & 5 & - & - \\
\hline$"$ & Monotagma densiflorum (Körn.) K. Schum. & $"$ & 5 & - & - & - \\
\hline
\end{tabular}




\section{ACTA \\ AMAZONICA}

ASPECTOS FLORÍSTICOS, FITOSSOCIOLÓGICOS E ECOLÓGICOS DE UM

SUB-BOSQUE DE TERRA FIRME NA AMAZÔNIA CENTRAL, AMAZONAS, BRASIL.

\begin{tabular}{|c|c|c|c|c|c|c|}
\hline Famílias & Nomes Científicos & Hábitos & C1 & C2 & C3 & C4 \\
\hline$"$ & Monotagma tuberosum Hagberg & $"$ & 86 & 14 & - & - \\
\hline$"$ & Monotagma sp. 1 & " & - & 6 & - & - \\
\hline " & Monotagma sp. 2 & $"$ & - & 2 & - & - \\
\hline Melastomataceae & Henriettella sp. & árvore & - & - & - & 2 \\
\hline " & Miconia affinis DC. * & " & 1 & - & - & - \\
\hline$"$ & M. argyrophylla DC. & " & 4 & - & - & - \\
\hline " & M. cf. comptifolia Wurdack & $"$ & 9 & 4 & - & - \\
\hline$"$ & M. tomentosa (Rich.) D. Don ex DC. * & $"$ & - & - & - & 1 \\
\hline Meliaceae & Guarea carinata Ducke* & $"$ & - & 1 & - & - \\
\hline " & G. cinnamomea Harms & $"$ & - & 1 & 1 & - \\
\hline Memecylaceae & Mouriri duckeana Morley & " & 2 & - & - & - \\
\hline$"$ & M. lunatanthera Morley* & $"$ & - & - & 1 & - \\
\hline$"$ & Mouriri sp. & " & 2 & - & - & - \\
\hline Menispermaceae & Abuta brevifolia Krukoff \& Moldenke* & liana & 1 & - & - & - \\
\hline " & A. candollei Triana \& Planch. & " & 8 & - & 2 & 1 \\
\hline$"$ & A. velutina Gleason* & $"$ & 1 & - & - & - \\
\hline Mimosaceae & Abarema laeta (Benth.) Barneby \& Grimes & árvore & 6 & 1 & - & 2 \\
\hline$"$ & Inga alba (Swart.) Willd. & $"$ & 3 & 2 & - & 2 \\
\hline$"$ & I. auristellae Harms & $"$ & 8 & - & - & - \\
\hline$"$ & I. bicoloriflora Ducke & " & 41 & 3 & 2 & - \\
\hline$"$ & I. capitata Desv. & $"$ & 5 & 2 & - & - \\
\hline$"$ & I. cf. nobilis Willd. & $"$ & - & 3 & - & 1 \\
\hline$"$ & I. cf. splendens Willd. & $"$ & 3 & - & - & - \\
\hline$"$ & I. chrysantha Ducke* & árvore & 1 & - & - & - \\
\hline$"$ & I. ciliata C. Presl. * & $"$ & 1 & - & - & - \\
\hline$"$ & I. edulis Mart. * & $"$ & - & 1 & - & - \\
\hline$"$ & I. grandiflora Wall. & $"$ & 6 & - & 1 & - \\
\hline " & I. huberi Ducke* & " & 1 & - & - & - \\
\hline " & I. leiocalycina Benth. & " & - & 3 & - & 1 \\
\hline$"$ & I. obidensis Ducke & $"$ & 10 & 2 & 3 & - \\
\hline$"$ & I. paraensis Ducke & " & 5 & - & - & 1 \\
\hline$"$ & I. umbratica Poepp. \& Endl. & $"$ & 4 & 2 & - & - \\
\hline$"$ & Mimosa guilandinae (DC) Barneby & liana & 2 & - & - & - \\
\hline$"$ & Parkia cf. decussata Ducke* & árvore & - & 1 & - & - \\
\hline$"$ & P. pendula (Willd.) Walp. & " & 2 & - & - & 1 \\
\hline$"$ & Stryphnodendron sp. * & $"$ & - & - & - & 1 \\
\hline " & Zygia racemosa (Ducke) Barbeby \& Grimes & " & 4 & - & 1 & 1 \\
\hline$"$ & Z. ramiflora (Benth.) Barneby \& Grimes & " & 45 & 1 & 4 & 2 \\
\hline Monimiaceae & Bracteanthus glycycarpus Ducke* & arbusto & 1 & - & - & - \\
\hline Moraceae & Brosimum acutifolium Huber & árvore & 3 & - & - & - \\
\hline$"$ & B. lactescens (S. Moore) C.C. Berg & $"$ & 5 & - & - & 1 \\
\hline$"$ & B. rubescens Taub. & " & 4 & 3 & - & - \\
\hline$"$ & Clarisia racemosa Ruiz \& Pav. & $"$ & 7 & 3 & - & - \\
\hline$"$ & Helianthostylis sprucei Baill. & árvore & 9 & 2 & - & 1 \\
\hline$"$ & Helicostylis sp. & $"$ & 6 & - & - & - \\
\hline$"$ & Maquira calophylla (Poepp. \& Endl.) C.C. Berg & $"$ & 5 & 1 & - & - \\
\hline " & Naucleopsis caloneura (Huber) Ducke & " & 8 & - & - & - \\
\hline$"$ & Pseudolmedia laevis (Ruiz \& Pav.) Macbr. * & $"$ & - & 1 & - & - \\
\hline$"$ & Sorocea muriculata Miq. & $"$ & 2 & 4 & 1 & 1 \\
\hline
\end{tabular}




\section{ACTA \\ AMAZONICA}

\begin{tabular}{|c|c|c|c|c|c|c|}
\hline Famílias & Nomes Científicos & Hábitos & C1 & C2 & C3 & C4 \\
\hline Myristicaceae & Iryanthera coriacea Ducke* & $"$ & - & 1 & - & - \\
\hline " & Virola calophylla (Spruce) Warb. & $"$ & 4 & - & - & - \\
\hline$"$ & V. michelii Heckel & $"$ & 4 & - & 1 & - \\
\hline Myrsinaceae & Cybianthus sp. & $"$ & 6 & - & - & - \\
\hline Myrtaceae & Eugenia anastomosans DC. & $"$ & - & - & 2 & - \\
\hline$"$ & E. cf. dittocrepis O. Berg & $"$ & - & 2 & - & 1 \\
\hline$"$ & E. patrisii Vahl & $"$ & 2 & - & - & - \\
\hline " & E. protenta Mc Vaugh & $"$ & 4 & - & - & - \\
\hline " & E. ramiflora Desv. & $"$ & - & 2 & 3 & - \\
\hline$"$ & Eugenia sp. & $"$ & - & 4 & - & - \\
\hline$"$ & Eugenia sp. nova* & $"$ & 1 & - & - & - \\
\hline$"$ & Myrcia amazonica DC. * & $"$ & 1 & - & - & - \\
\hline$"$ & M. cf. bracteata (Rich.) DC. & $"$ & - & 1 & 1 & - \\
\hline$"$ & M. cf. eximia DC. & $"$ & 2 & - & - & - \\
\hline$"$ & M. fenestrata DC. & $"$ & 1 & - & - & 1 \\
\hline " & M. magnoliifolia DC. & " & 2 & - & 1 & 1 \\
\hline Nyctaginaceae & Neea oppositifolia Ruiz \& Pav. & $"$ & 2 & 2 & - & 1 \\
\hline Ochnaceae & Ouratea discophora Ducke* & $"$ & - & 1 & - & - \\
\hline Olacaceae & Minquartia guianensis Aubl. & $"$ & - & 2 & - & 1 \\
\hline " & Aptandra tubicina (Poepp.) Benth. ex Miers* & $"$ & 1 & - & - & - \\
\hline$"$ & Heisteria sp. & $"$ & - & 3 & - & - \\
\hline$"$ & Ptychopetalum olacoides Benth. & $"$ & 2 & 1 & - & 1 \\
\hline Passifloraceae & Passiflora foroana Harms* & liana & 1 & - & - & - \\
\hline Piperaceae & Piper bartlingianum (Miq.) C. DC. & arbusto & 9 & - & - & - \\
\hline Poaceae & Pariana cf. campestris Aubl. & erva & 56 & 18 & - & - \\
\hline Polygonaceae & Coccoloba sp. & liana & 2 & 2 & - & 1 \\
\hline Quiinaceae & Lacunaria crenata (Tul.) A.C. Sm. & árvore & 2 & - & - & - \\
\hline " & Quiina amazonica A.C. Sm. & $"$ & 2 & - & - & - \\
\hline$"$ & Quiina sp. * & $"$ & - & - & 1 & - \\
\hline$"$ & Touroulia guianensis Aubl. & $"$ & 2 & - & - & - \\
\hline Rhabdodendraceae & Rhabdodendron amazonicum (Spruce ex Benth.) Huber & $"$ & - & 3 & - & - \\
\hline Rhamnaceae & Ampelozizyphus amazonicus Ducke & liana & 3 & 3 & 1 & - \\
\hline Rubiaceae & Alibertia sp. * & árvore & 1 & - & - & - \\
\hline " & Amaioua sp. * & $"$ & - & - & 1 & - \\
\hline$"$ & Duroia hirsuta (Poepp.) K. Schum. & $"$ & 4 & - & 1 & - \\
\hline$"$ & D. macrophylla Huber & $"$ & 3 & - & - & - \\
\hline$"$ & D. saccifera (Mart.) Hook. f. ex K. Schum. ${ }^{*}$ & $"$ & - & - & - & 1 \\
\hline$"$ & Duroia sp. * & $"$ & - & 1 & - & - \\
\hline$"$ & Faramea sp. & $"$ & 1 & 2 & - & 1 \\
\hline$"$ & Palicourea comitis (Müll. Arg.) Steyerm. & $"$ & 4 & 3 & 2 & - \\
\hline$"$ & Palicourea sp. & $"$ & - & 2 & - & - \\
\hline$"$ & Psychotria astrellantha Wernh. & árvore & 6 & 2 & - & 3 \\
\hline$"$ & P. bahiensis DC. * & arbusto & 1 & - & - & - \\
\hline$"$ & P. bracteocardia (DC.) Müll. Arg. * & $"$ & 1 & - & - & - \\
\hline$"$ & P. deinocalix Sandwith & $"$ & 2 & - & - & - \\
\hline$"$ & P. platypoda DC. & $"$ & 3 & - & - & - \\
\hline$"$ & P. podocephala Standl. & $"$ & 4 & 12 & 1 & - \\
\hline$"$ & Psychotria sp. * & $"$ & 1 & - & - & - \\
\hline " & Sabicea amazonensis Wernham* & liana & 1 & - & - & - \\
\hline
\end{tabular}




\section{ACTA AMAZONICA}

\begin{tabular}{|c|c|c|c|c|c|c|}
\hline Famílias & Nomes Científicos & Hábitos & C1 & C2 & $\mathrm{C3}$ & C4 \\
\hline Sapindaceae & Cupania sp. * & árvore & - & - & - & 1 \\
\hline$"$ & Paullinia sp. & liana & 5 & - & - & - \\
\hline$"$ & Serjania membranacea Splitg. & $"$ & 9 & - & - & - \\
\hline " & Talisia cf. macrophylla (Mart.) Radlk. * & árvore & - & - & - & 1 \\
\hline$"$ & Talisia sp. * & $"$ & - & - & - & 1 \\
\hline$"$ & Toulicia sp. & $"$ & 4 & - & - & - \\
\hline Sapotaceae & Chrysophyllum amazonicum T.D. Penn. & $"$ & 3 & 1 & - & - \\
\hline$"$ & Chrysophyllum sp. & $"$ & - & - & - & 1 \\
\hline$"$ & Ecclinusa guianensis Eyma & $"$ & 5 & 2 & 2 & - \\
\hline$"$ & Manilkara huberi (Ducke) A.Chev. * & $"$ & 1 & - & - & - \\
\hline$"$ & Micropholis guyanensis (A.DC.) Pierre & $"$ & - & 1 & - & 1 \\
\hline$"$ & M. mensalis (Baehni) Aubrév. & $"$ & 9 & - & - & - \\
\hline$"$ & M. venulosa (Mart. \& Eichler) Pierre & $"$ & - & 2 & - & - \\
\hline$"$ & Pouteria anomala (Pires) T.D. Penn. & $"$ & 4 & - & - & 1 \\
\hline$"$ & P. cf. retinervis T.D. Penn. & $"$ & - & - & 2 & - \\
\hline$"$ & P. cuspidata (A.DC.) Baehni* & $"$ & - & - & - & 1 \\
\hline$"$ & P. decorticans T.D. Penn. & $"$ & 8 & - & - & - \\
\hline$"$ & P. durlandii (Standl.) Baehni * & $"$ & - & 1 & - & - \\
\hline$"$ & P. glomerata (Miq.) Baehni & $"$ & 7 & - & - & - \\
\hline$"$ & P. gongrijpii Eyma & $"$ & - & - & 2 & - \\
\hline$"$ & P. guianensis Aubl. & $"$ & 3 & 3 & - & 1 \\
\hline$"$ & P. macrophylla (Lam.) Eyma & $"$ & - & 3 & - & - \\
\hline$"$ & Pouteria sp. & $"$ & 1 & 1 & 1 & - \\
\hline Selaginellaceae & Selaginella conduplicata Spring. & erva & 5 & - & - & - \\
\hline Simaroubaceae & Simarouba amara Aubl. * & árvore & - & - & - & 1 \\
\hline Siparunaceae & Siparuna cuspidata (Tul.) A. DC. & $"$ & 5 & - & - & 1 \\
\hline$"$ & S. guianensis Aubl. & $"$ & 5 & 2 & - & - \\
\hline Solanaceae & Solanum sp.* & arbusto & 1 & - & - & - \\
\hline Sterculiaceae & Sterculia excelsa Mart. * & árvore & 1 & - & - & - \\
\hline$"$ & Theobroma silvestre Mart. * & $"$ & - & - & - & 1 \\
\hline Theophrastaceae & Clavija sp. * & arbusto & - & 1 & - & - \\
\hline Tiliaceae & Indeterminada* & árvore & 1 & - & - & - \\
\hline Ulmaceae & Trema micrantha (L.) Blume & árvore & 3 & 4 & - & 1 \\
\hline Verbenaceae & Petrea volubilis $\mathrm{L}$. & liana & 1 & 3 & 2 & - \\
\hline Violaceae & Amphirrhox longifolia Spreng. & arbusto & 2 & - & - & - \\
\hline$"$ & Leonia sp.* & árvore & - & - & - & 1 \\
\hline$"$ & Paypayrola grandiflora Tul. & $"$ & 15 & 9 & 2 & - \\
\hline$"$ & Rinorea cf. riana Kuntze & $"$ & 3 & 2 & - & - \\
\hline$"$ & R. flavescens (Aubl.) Kuntze & $"$ & - & - & 3 & 3 \\
\hline$"$ & R. guianensis Aubl. & $"$ & 2 & 1 & - & - \\
\hline$"$ & R. paniculata (Mart.) Kuntze & $"$ & 2 & 2 & - & - \\
\hline$"$ & R. racemosa (Mart.) Kuntze & " & 4 & 1 & 1 & 4 \\
\hline
\end{tabular}

Dos 2.434 indivíduos amostrados, 1471 ordenam-se em apenas dez famílias botânicas. Juntas, Marantaceae (209), Chrysobalanaceae (198), Mimosaceae (191), Burseraceae (175), Annonaceae (172), Arecaceae (137), Caesalpiniaceae (114), Fabaceae (100), Lecythidaceae (92) e, por fim, Bignoniaceae (83) representam mais de $60 \%$ desse total; os outros 963 indivíduos distribuem-se entre as outras 57 famílias, evidenciando, com isso, alta densidade em poucas famílias botânicas, conforme observado em outras comunidades de terra firme na Amazônia Central (Lima Filho, 1995; Amaral et al., 2000; Lima Filho et al., 2002). 


\section{ACTA \\ AMAZONICA}

ASPECTOS FLORÍSTICOS, FITOSSOCIOLÓGICOS E ECOLÓGICOS DE UM

SUB-BOSQUE DE TERRA FIRME NA AMAZÔNIA CENTRAL, AMAZONAS, BRASIL.
Analisando o número de indivíduos por espécie, notou-se que apenas seis espécies detêm 588 indivíduos, quase $25 \%$ do total de espécimes registrados. Dentre as espécies mais abundantes, Licania caudata Prance contribui com 145 indivíduos (Tabela 1), o que corresponde a $6 \%$ da densidade total amostrada. Estudo desenvolvido em uma floresta de terra firme no rio Uatumã, Amazonas (Amaral et al., 2000) revelou que, no estrato inferior da floresta, as espécies Protium apiculatum Swart e Pariana sp. figuram entre as cinco mais expressivas em número de indivíduos, corroborando os resultados encontrados nesse trabalho (Tabelas 1 e 3).

O percentual de espécies "localmente raras" (Oliveira $e t$ al., dados não publicados) na amostragem foi de 48\% (169), dentro do intervalo de 25 a $56 \%$ relatado para as espécies com $\mathrm{DAP} \geqslant 10 \mathrm{~cm}$, nos diversos trabalhos desenvolvidos na região (Porto et al., 1976; Tello, 1995; Amaral, 1996). Para algumas espécies, a raridade pode atuar como um mecanismo biológico de defesa contra a ação de predadores (Viana et al., 1992); os indivíduos esparsos formam populações que ocupam grandes áreas, podendo obter com isso maior êxito na perpetuação da espécie. Por outro lado, as espécies raras podem tornar-se mais vulneráveis à extinção porque, em geral, são especializadas a um conjunto restrito de fatores ambientais ou têm poderes limitados de se dispersar para outras áreas.

A família Fabaceae apresentou o maior número de espécies (27), seguida por Mimosaceae (22), Lauraceae (21), Caesalpiniaceae e Rubiaceae (18), Sapotaceae (17), Euphorbiaceae e Myrtaceae (12), Arecaceae, Chrysobalanaceae e Lecythidaceae (11) (Figura 2). Conjuntamente, essas onze famílias representam mais de $50 \%$ da riqueza local, assemelhando-se aos padrões registrados em outros estudos de regeneração natural na Amazônia Central (Vieira, 1989; Lima Filho, 1995; Lima Filho et al., 2002).

Ainda com relação à riqueza de espécies, Euphorbiaceae foi a que apresentou a maior relação entre o número de espécies "localmente raras" e o número de espécies identificadas, istoé, a cada três espécies identificadas dessa família, duas foram raras (2/ 3). Em seguida, surgem as famílias Rubiaceae e Caesalpiniaceae com proporções de $1 / 2$ e 4/9, respectivamente (Tabelas 1 e 2 ). Por outro lado, Arecaceae foi a única família na qual encontrou-se mais de um indivíduo por espécie, portanto, não registrando-se nessa amostragem espécies "localmente raras". De qualquer forma, esses resultados demonstram a importância dessas espécies como um dos fatores responsáveis pela alta fitodiversidade nas florestas amazônicas, conforme sugerido em outros trabalhos concluídos na região (Lima Filho, 1995; Tello, 1995; Amaral, 1996; Matos \& Amaral, 1999; Amaral et al., 2000; Lima Filho et al., 2002).

\section{Distribuição espacial}

Analisando-se a distribuição espacial das 30 espécies com maior participação na estrutura vertical da fitocenose (Tabela 3), observou-se que apenas Astrocaryum gynacanthum Mart. encontra-se aleatoriamente distribuída na área amostral. A tendência de distribuição espacial, em que a distribuição aleatória é rara em populações vegetais, sendo que a maioria das espécies possui algum grau de agregação no espaço, tem sido constatada em outros estudos (Legendre \& Fortin, 1989; Horvitz \& Le Corff, 1993). As espécies com maiores índices de agregação foram Licania caudata Prance, Zygia ramiflora (Benth.) Barneby \& Grimes, Sclerolobium sp. 1, Lindsaea lancea (L.) Bedd. e Ocotea longifolia Kunth (Tabela 4).

\section{Diversidade florística}

A curva espécie-área indicou que a comunidade vegetal é bastante diversificada em termos florísticos; o modelo matemático que melhor explicou os dados foi o polinomial de quarto grau (Figura 3), através do qual foi possível observar um sinal inicial de saturação da curva (paralelo ao eixo X) já a partir da última parcela. Assim, a partir da equação, foi possível estimar 357 espécies contra 355 espécies encontradas. Portanto, essas informações permitem inferir que a amostragem de 20 parcelas de $5 \times 5 \mathrm{~m}$ foram suficientes para estimar a riqueza de espécies da fitocenose.

Ainda com relação à curva espécie-área, vale ressaltar que esta sofreu grande influência da seqüência em que as parcelas foram alocadas, pois a riqueza florística foi muito variável entre as parcelas (Figura 3). O número de espécies por parcela oscilou de 5 a 46, com média de 18 espécies $/ 25 \mathrm{~m}^{2}$ e desvio padrão de 11,2 , com as parcelas iniciais apresentando as maiores concentrações de espécies.

Quanto aosíndices de diversidade de Shannon ( $\left.\mathrm{H}^{\prime}\right)$, os valores variaram de 2,83 a 5,25 nats/indivíduo entre as quatro classes de altura (Tabela3). As maiores riquezas e diversidades encontradas nas classes 1 (altura $£ 0,5 \mathrm{~m})$ e $2(0,5<$ altura $\leqslant$ $1,5 \mathrm{~m})$ de altura eram previsíveis para a fitocenose, já que, naturalmente, muitas espécies deervas, arbustose lianas herbáceas são amostradas apenas nas classes inferiores dealtura. Éintrigante, no entanto, a ausência nessas mesmas classes de 12 espécies arbóreas ordenadas entre as 20 de maior importânciaecológica nafitocenose (Oliveira et al., dados não publicados), algumas das quaisencontradas emalta densidadenaárea, como: Pouteria gongrippii Eyma, Chrysophyllum sanguinolentum (Pierre) Baehni,Eschweilera atropetiolata S.A. Mori, 


\section{ACTA AMAZONICA}

Tabela 3 - Listagem e parâmetros quantitativos da estrutura vertical das espécies vegetais presentes no sub-bosque de uma floresta de terra firme na Amazônia Central, Amazonas, Brasil. Onde: RNT = regeneração natural total, RNR = regeneração natural relativa, PSA = posição sociológica absoluta, PSR = posição sociológica relativa, PDE = padrão de distribuição espacial.

\begin{tabular}{|c|c|c|c|c|c|c|c|}
\hline Famílias & Nomes Científicos & $\mathrm{Ni}$ & RNT & RNR & PSA & PSR & PDE \\
\hline Chrysobalanaceae & Licania caudata Prance & 145 & 14,43 & 4,78 & 92,63 & 8,20 & agregado \\
\hline Annonaceae & Duguetia flagellaris Huber & 126 & 10,95 & 3,65 & 40,23 & 3,56 & agregado \\
\hline Marantaceae & Monotagma tuberosum Hagberg & 100 & 10,47 & 3,49 & 58,62 & 5,19 & agregado \\
\hline Burseraceae & Protium apiculatum Swart & 91 & 9,72 & 3,24 & 48,72 & 4,32 & agregado \\
\hline Poaceae & Pariana cf. campestris Aubl. & 74 & 7,80 & 2,60 & 40,02 & 3,54 & agregado \\
\hline Mimosaceae & Zygia ramiflora (Benth.) Barneby \& Grimes & 52 & 5,19 & 1,73 & 29,41 & 2,61 & agregado \\
\hline Mimosaceae & Inga bicoloriflora Ducke & 46 & 5,19 & 1,73 & 27,20 & 2,41 & agregado \\
\hline Caesalpiniaceae & Sclerolobium sp. 1 & 40 & 4,14 & 1,38 & 23,51 & 2,08 & agregado \\
\hline Arecaceae & Oenocarpus bacaba Mart. & 37 & 4,80 & 1,60 & 21,61 & 1,91 & agregado \\
\hline Marantaceae & Ischnosiphon cannoideus L. Andersson & 32 & 3,09 & 1,03 & 13,53 & 1,20 & agregado \\
\hline Dennstaedtiaceae & Lindsaea lancea (L.) Bedd. & 32 & 3,45 & 1,15 & 20,73 & 1,84 & agregado \\
\hline Lecythidaceae & Eschweilera bracteosa (Poepp. ex O.Berg) Miers & 28 & 3,24 & 1,08 & 15,58 & 1,38 & agregado \\
\hline Heliconiaceae & Heliconia acuminata Rich. & 28 & 3,21 & 1,07 & 15,50 & 1,37 & agregado \\
\hline Burseraceae & Protium divaricatum Engl. & 28 & 2,97 & 0,99 & 12,74 & 1,13 & agregado \\
\hline Bignoniaceae & Arrabidaea egensis Bureau \& K. Schum. & 28 & 3,36 & 1,12 & 12,02 & 1,06 & agregado \\
\hline Burseraceae & Protium hebetatum Daly & 27 & 2,64 & 0,88 & 11,85 & 1,05 & agregado \\
\hline Violaceae & Paypayrola grandiflora Tul. & 26 & 3,03 & 1,01 & 11,60 & 1,03 & agregado \\
\hline Lauraceae & Ocotea longifolia Kunth & 25 & 2,88 & 0,96 & 14,68 & 1,30 & agregado \\
\hline Marantaceae & Calathea mansonis Körn. & 22 & 2,61 & 0,87 & 14,25 & 1,26 & agregado \\
\hline Marantaceae & Ischnosiphon arouma (Aubl.) Körn. & 21 & 1,89 & 0,63 & 6,56 & 0,58 & agregado \\
\hline Euphorbiaceae & Mabea angularis Hollander & 21 & 2,19 & 0,73 & 5,56 & 0,49 & agregado \\
\hline Arecaceae & Astrocaryum gynacanthum Mart. & 21 & 2,70 & 0,90 & 5,64 & 0,50 & aleatório \\
\hline Arecaceae & Astrocaryum sciophilum (Miq.) Pulle & 20 & 2,46 & 0,82 & 7,95 & 0,70 & agregado \\
\hline Lecythidaceae & Eschweilera coriacea (DC.) S.A. Mori & 19 & 2,58 & 0,86 & 9,71 & 0,86 & agregado \\
\hline Connaraceae & Connarus erianthus Benth. ex Baker & 18 & 2,55 & 0,85 & 8,82 & 0,78 & agregado \\
\hline Caesalpiniaceae & Peltogyne paniculata Benth. & 18 & 2,16 & 0,72 & 11,02 & 0,98 & agregado \\
\hline Rubiaceae & Psychotria podocephala Standl. & 17 & 2,07 & 0,69 & 5,09 & 0,45 & agregado \\
\hline Bignoniaceae & Memora cf. magnifica (Mart. ex DC.) Bureau & 16 & 1,95 & 0,65 & 5,36 & 0,47 & agregado \\
\hline Mimosaceae & Inga obidensis Ducke & 15 & 1,71 & 0,57 & 6,91 & 0,61 & agregado \\
\hline Hippocrateaceae & Salacia insignis A.C. Sm. & 15 & 2,04 & 0,68 & 7,08 & 0,63 & agregado \\
\hline
\end{tabular}

Licania davillifolia Benoist., entre outras. Em ambientes florestais, a ausência de espécies heliófitas nas classes inferiores é até esperada. As espéciesque dependem de clareiras paragerminaremapresentam um padrão de dispersão sazonal, que precede a época de maior clareiras naturais que, no caso da Amazônia Central, coincide como período chuvoso, no qual as árvores são derrubadas pela ação do vento.Agora, poralguma razão, talvez relacionada aociclohidrológico ou a outros fatores associados à biologia reprodutiva das espécies ou ao próprio ambiente, vem dificultando o recrutamento de novos indivíduos à comunidade.

Excetuando-se a classe 4 (altura $>3$ me CAP $<0,3 \mathrm{~m}$ ), os dados mostram decréscimos na diversidade da menor para a maior categoria de altura (Tabela 3). De acordo com alguns autores (Odum, 1983; Magurran, 1988; Kent \& Coker, 1992), a diversidadebiológica envolve dois componentes básicos: a riqueza em espécies e a uniformidade na distribuição das abundâncias na comunidade. Diante disso, é possível que a exceção observada deva-se ao maior número de espécies encontrada nessa amplitude de tamanho em relação à classe $3(1,5<$ altura $\leqslant 3,0 \mathrm{~m})$, apesar de possuírem a mesma distribuição dentro de suas respectivas populações (Tabela 3).

O H' para a área total foi de 5,60 nats/indivíduo (Tabela 3), indicando que esse ambiente florestal apresenta grande diversidade florística, pois suplantou os limites máximos de 4,5 a 5,0 nats/indivíduo para as comunidades naturais (Pielou, 1966; Margalef, 1972). Essa alta diversidade pode ser confirmada ainda a partir da curva espécie-área (Figura 3) .

Quando comparada a diversidade obtida nesse estudo às de outras florestas brasileiras (Gisler, 1995; Nappo, 1999), cujos índices variaram de 2,7 a 4,1 para a regeneração natural, pode-se concluir que a área inventariada é uma das mais diversificadas para esses grupos de vegetação.

Quanto aos índices de equabilidade de Shannon (E') por categoria de altura, nota-se que houve maior uniformidade 


\section{ACTA AMAZONICA}

ASPECTOS FLORISTICOS, FITOSSOCIOLÓGICOS E ECOLÓGICOS DE UM SUB-BOSQUE DE TERRA FIRME NA AMAZÔNIA CENTRAL, AMAZONAS, BRASIL. na primeira categoria (Tabela 3) com 0,95, indicando que as espécies jovens tendem a distribuírem-se mais uniformemente dentro da comunidade vegetal.

Por outro lado, os graus de E' obtidos para as espécies das demais categorias de altura sugerem que a distribuição dessas plantas dentro da comunidade é apenas moderadamente uniforme. De qualquer forma, o grau de E' estimado para a área amostrada indica que a distribuição das espécies dentro da fitocenose é bastante uniforme, uma vez que esse valor aproximou-se de 1 (Margalef, 1958).

\section{Similaridade florística}

A análise de similaridade entre as classes de altura mostraram maior similaridade florística entre classes 1 e 2 (Tabela 3). O coeficiente de 0,50 calculado entre as classes 1 e 2 indica que, caso uma espécie seja aleatoriamente sorteada, ela possui $50 \%$ de probabilidade de pertencer às duas classes de altura. Por outro lado, todas as combinações feitas com a classe 3 resultaram nos menores índices de similaridade (Tabela 3), possivelmente associados à menor diversidade de espécies nessa categoria de altura (Tabela 4).

Quando as espécies "localmente raras" foram excluídas da análise, registrou-se aumento médio de $21 \%$ na similaridade entre as categorias avaliadas (Tabela 4). Esse resultado sugere que a similaridade florística entre comunidades vegetais pode estar associada, pelo menos em parte, ao número de espécies "localmente raras" presentes na amostragem, conforme observado para as espécies arbóreas (Oliveira et al., dados não publicados).

\section{Estrutura da regeneração natural e do sub-bosque}

Dentre as 355 espécies identificadas no levantamento, 30 destacaram-se relativamente quanto aos parâmetros regeneração natural e posição sociológica, retratandocerca de $43 \%$ de todas as espécies constantes na amostragem (Tabela 3). Notou-se ainda, que, desse percentual, as espécies Licania caudata Prance, Duguetiaflagellaris Huber, Monotagma tuberosum Hagberg, Protium apiculatum Swart, ePariana cf. campestris Aubl., respondem por $18 \%$ da regeneração natural. Enquanto isso, 283 espécies obtiveram índices de regeneração inferiores a 1,0 , representando em sua totalidade mais de $38 \%$ das espécies registradas.

A partir das densidades relativas das plantas em regeneração, ponderou-se que dentre as mais significativas, Licania caudata Prance, Duguetia

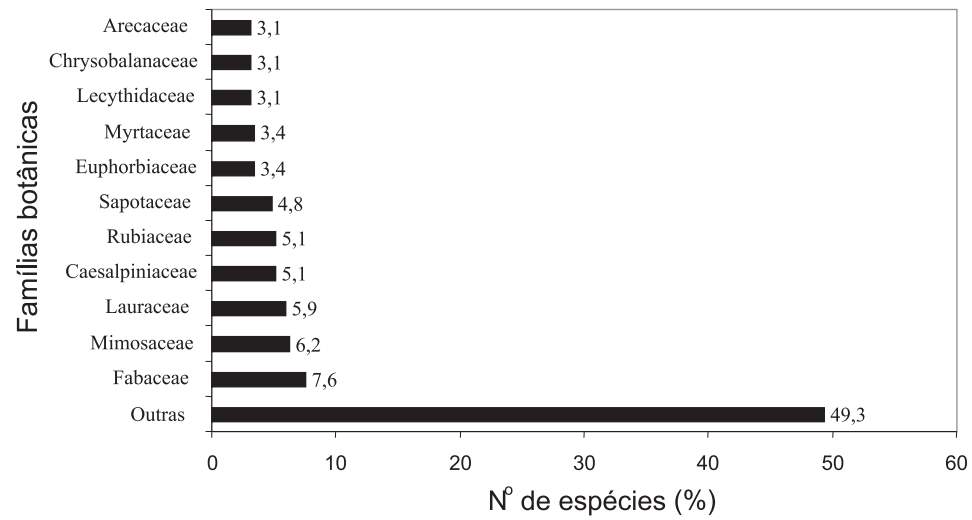

Figura 2 - Percentuais de espécies por família nos grupos de vegetação de regeneração natural e sub-bosque de uma floresta de terra firme na Amazônia Central, Amazonas, Brasil.

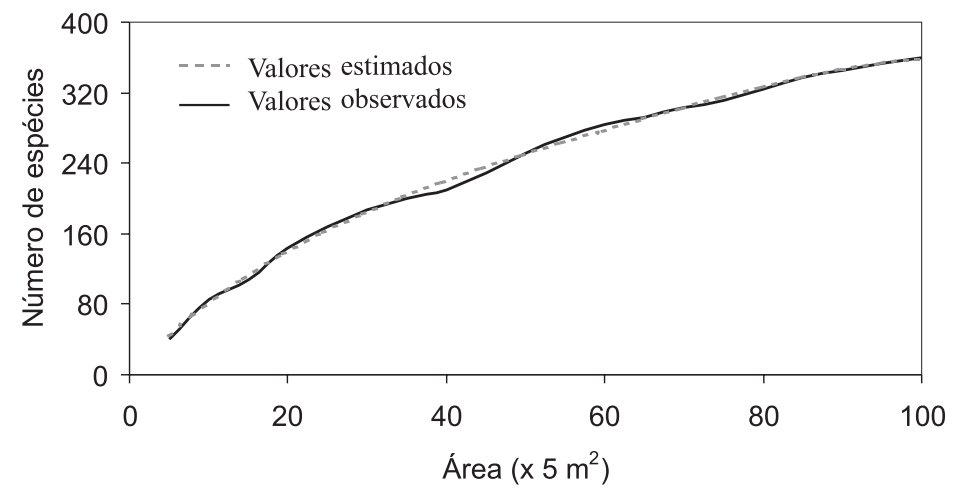

Figura 3 - Curva cumulativa das espécies constante no sub-bosque de uma floresta de terra firme na Amazônia Central, Amazonas, Brasil.

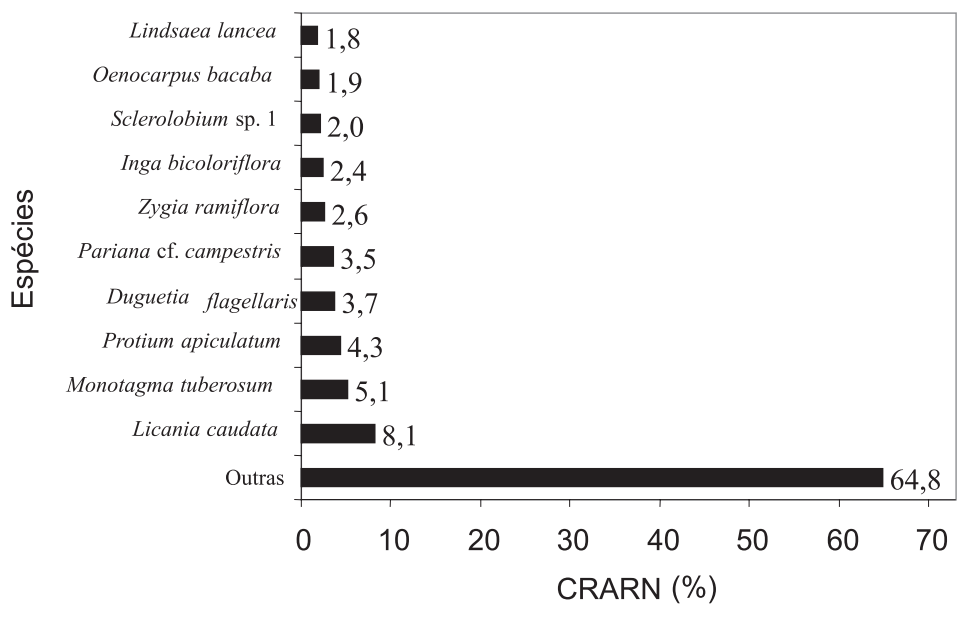

Figura 4 - Classes relativas de altura da regeneração natural (CRARN) das dez espécies mais importantes de um sub-bosque de terra firme na Amazônia Central, Amazonas, Brasil 


\section{ACTA AMAZONICA}

Tabela 4 - Índices de similaridade de Sorensen entre as quatro classes de altura estudadas. Amazônia Central, Amazonas, Brasil.

\begin{tabular}{|c|c|c|c|c|c|c|c|c|}
\hline \multirow[b]{2}{*}{ Classes de altura } & \multicolumn{4}{|c|}{$\begin{array}{c}\text { Com todas as } \\
\text { espécies identificadas }\end{array}$} & \multicolumn{4}{|c|}{$\begin{array}{l}\text { Sem as espécies } \\
\text { "localmente raras" }\end{array}$} \\
\hline & $\mathrm{C} 1$ & $\mathrm{C} 2$ & $\mathrm{C} 3$ & $\mathrm{C} 4$ & C1 & $\mathrm{C} 2$ & $\mathrm{C} 3$ & $\mathrm{C} 4$ \\
\hline$\overline{\mathrm{C} 1}$ & & 0,50 & 0,35 & 0,42 & & 0,61 & 0,42 & 0,52 \\
\hline $\mathrm{C} 2$ & & & 0,39 & 0,45 & & & 0,46 & 0,54 \\
\hline $\mathrm{C} 3$ & & & & 0,37 & & & & 0,45 \\
\hline $\mathrm{C} 4$ & & & & & & & & \\
\hline
\end{tabular}

ecológica das espécies dentro da comunidade vegetal.

Comparando as duas espécies mais representativas da regeneração natural, nota-se que apenas dois dos 145 indivíduos de Licania caudata Prance encontram-se na classe 2 de altura (Tabela 1). Ao passo que os 126 indivíduos da espécie Duguetia flagellaris Huber distribuem-se uniformemente em todas as classes abordadas (Tabela 3). Essa constatação permite sugerir que nesse

flagellaris Huber, Monotagma tuberosum Hagberg, Protium apiculatum Swart e Pariana cf. campestris Aubl., compõem as cinco mais expressivas da amostragem (Tabela 3), contribuindo em conjunto com $22 \%$ da área total.

Quanto às freqüências relativas, as espécies de maior destaque foram Duguetia flagellaris Huber, Protium apiculatum Swart, Oenocarpus bacaba Mart., Astrocaryum gynacanthum Mart., Pariana cf. campestris Aubl., Monotagma tuberosum Hagberg, Arrabidaea egensis Bureau \& K. Schum., e Connarus erianthus Hagberg. Tais resultados assemelham-se aos encontrados por outros autores (Higuchi et al., 1985; Lima Filho, 1995; Lima-Filho et al., 2002) em florestas de terra firme na Amazônia Central. Por outro lado, as demais espécies detiveram freqüências relativas abaixo de $1 \%$, incluindo Licania caudata Prance que na amostragem respondeu por apenas $0,35 \%$. A baixa freqüência observada para essa espécie já era esperada, pois seus indivíduos encontram-se agrupados em manchas na amostragem (chuva de sementes), isto é, das 20 parcelas avaliadas nesse estudo, a ocorrência dessa espécie resume-se a apenas três, oque pode comprometer significativamente sua propagação e dispersão na floresta.

Os níveis relativos obtidos para as categorias de altura da regeneração mostram que, dentre as espécies analisadas, Licania caudata Prance, Monotagma tuberosum Hagberg, Protium apiculatum Swart, Duguetia flagellaris Huber e Pariana cf. campestris Aubl. detêm os maiores índices para este parâmetro estrutural (Figura 4). Em contrapartida, 14 espécies variaram de 1,0 a 2,6\%, e o restante delas ficou abaixo de $1 \%$.

Segundo alguns estudos (Volpato, 1994; Nappo, 1999), a metodologia proposta por Finol (1971) para estimar a regeneração natural induz as espécies que possuem alta densidade em uma determinada classe de altura, a manifestarem os maiores valores para esse parâmetro vertical. Esses estudos podem ser demonstrados por intermédio de Licania caudata Prance e Monotagma tuberosum Hagberg que apresentaram, respectivamente, 145 e 86 indivíduos, todos na classe inferior de altura (Tabela 1), gerando os dois maiores valores relativos de altura da regeneração natural (CRARN) (Figura 4). A partir dessas informações, verificou-se que os maiores índices de CRARN nem sempre significam maiores contribuições na regeneração natural total, uma vez que Duguetia flagellaris Huber, embora tenha o quarto maior CRARN, foi a segunda em participação na regeneração natural das demais espécies (Tabela3). Diante disso, pode-se inferir que os valores de regeneração natural alto, a partirda CRARN também alto, podem não retratar de forma confiável a real importância momento e nas condições ambientais avaliadas, Duguetia flagellaris Huber é a mais importante em relação às outras espécies, por estar melhor distribuída na área e por ocupar todas as categorias de desenvolvimento das espécies em regeneração.

Quando se analisa a abundância de lianas e palmeiras na regeneração natural das demais espécies de plantas, verifica-se que essas formas de vida contribuem com 5,6 e 5,9\%, respectivamente, evidenciando suas importâncias dentro da comunidade estudada, bem como para a análise estrutural desse tipo de vegetação, conforme observado e discutido em outros estudos na região (Jardim, 1985; Lima Filho, 1995).

A classe de altura dominante em termos quanti-qualitativos foi a $\mathrm{C} 1$ (altura $\leqslant 0,5 \mathrm{~m}$ ), com 1.583 indivíduos, classificados em 59 famílias, 125 gênerose 247 espécies (Tabela 4). Essa tendência, em que o número de espécimes na classe de menor altura prevalece sobre as demais, tem sido relatada em outros estudos conduzidos na região (Vieira, 1989; Lima Filho, 1995; Matos \& Amaral, 1999; Amaral et al., 2000). Uma possível explicação para este fato seria o grande número de indivíduos que durante todo o seu ciclo de vida jamais atingirão outras classes de altura, como as ervas, arbustos e algumas lianas (Tabela 1). Ou ainda, por se tratar de uma floresta nativa, o nível de perturbação ambiental é mínimo, não acarretando danos maiores ao estabelecimento e colonização da área por essas plantas.

\section{BIBLIOGRAFIA CITADA}

Amaral, I.L. 1996. Diversidade Florística em Floresta de Terra Firme, na região do rio Urucu- AM. Dissertação de Mestrado. INPA/UFAM. Manaus, AM. 160pp.

Amaral, I.L.; Matos, F.D.A.; Lima, J. 2000. Composição florística e estrutural de um hectare de floresta densa de terra firme no Rio Uatumã, Amazônia, Brasil. Acta Amazonica, 30:377-392.

Carvalho, J.O.P. 1987. Subsídios para o manejo de florestas naturais na Amazônia Brasileira: Resultados de pesquisas da AMBRAPA/IBDF - PNF. Belém, EMBRAPA/CPATU. 35pp. (EMBRAPA/CPATU. Documentos, 43).

Chauvel, A.; Lucas, Y.; Boulet, R. 1987. On the genesis of the soil mantle of the region of Manaus, Central Amazonia, Brazil. Experientia, 43:234-241.

Ferraz, J.; Ohta, S.; Sales, P.C. 1998. Distribuição dos solos ao longo de dois transectos em floresta primária ao Norte de Manaus (AM). In: Higuchi, N.; Campos, M.A.A.; Sampaio, 
P.T.B.; Santos, J. (Eds.). Pesquisas florestais para a conservação da floresta e reabilitação de áreas degradadas da Amazônia. Manaus: INPA. p. 111-143.

Finol, U.H. 1971. Nuevos parametros a considerarse em el analisis estrutural de las selva virgenes tropicales. Rev. For. Venezolana, 14:29-42.

Gentry, A.H., Dodson, C. 1987. Diversity and phytogeografy of Neotropical epiphytes. Ann. Mo. Bot. Gard., 74:205-233.

Gisler, V.T. 1995. O uso da serrapilheira na recomposição da cobertura vegetal em áreas mineradas de Bauxita, Poços de Caldas, MG. Dissertação de Mestrado. USP. São Paulo, SP. 147pp.

Higuchi, N.; Jardim, F.C.S.; Santos, J.; Alencar, J.C. 1985. Bacia 3 - Inventário Diagnóstico da Regeneração Natural. Acta Amazonica, 15:199-233.

Higuchi, N.; Santos, J.; Vieira, G.; Ribeiro, R.J.; Sakurai, S.; Ishizuca, M.; Sakai, T.; Tanaka, N.; Saito, S. 1998. Análise Estrutural da Floresta Primária da Bacia do Rio Cuieiras, ZF-2, Manaus - AM, Brasil. In: Higuchi, N.; Campos, M.A.A.; Sampaio, P.T.B.; Santos, J. (Eds.). Pesquisas florestais para a conservação da floresta e reabilitação de áreas degradadas da Amazônia. Manaus: INPA. p. 53-81.

Horvitz, C.C.; Le Corff, J. 1993. Spatial scale and dispersion pattern of ant bird-herbs in two tropical lowland rain forests. Vegetatio, 107:351-362.

Jardim, F.C.S. 1985. Estrutura da floresta equatorial úmida da estação experimental de Silvicultura Tropical do INPA. Manaus - AM. Dissertação de Mestrado. INPA/UFAM. Manaus, AM. 195 pp.

Jardim, F.C.S.; Hosokawa, R.T. (1986/87). Estrutura da floresta equatorial úmida da Estação Experimental de Silvicultura Tropical do INPA. Acta Amazonica, 16/17: 411-508.

Kent, M.; Coker, P. 1992. Vegetation descripition and analysis, a practical approach. London: Belhaven Press. 263 pp.

Krebs C.J. 1989. Ecological methodology. Harper \& Row, New York, USA. 654 pp.

Lamprecht, H. 1964. Ensayo sobre la estrutura floristica de la parte suroriental del Bosque Universitário: "El caimital", Estado Barinas. Rev. For. Venezolana, 7:77-119.

Legendre, P.; Fortin, M.J. 1989. Spatial pattern and ecological analysis. Vegetatio, 80:107-138.

Lima Filho, D.A. 1995. Caracterização florística de 3 bectares de floresta de terra firme na região do rio Urucu. Dissertação de Mestrado. INPA/UFAM. Manaus, AM. 162 pp.

Lima-Filho, D.A; Revilla, J.; Coêlho, L.S.; Ramos, J.F.; Santos, J.L.; Oliveira, J.G. 2002. Regeneração natural de três hectares de floresta ombrófila densa de terra firme na região do rio Urucú, Amazonas, Brasil. Acta Amazonica, 32:555-570.

Magurran, A.E. 1988. Ecological diversity and its measurement. Princeton University Press, New Jersey, USA. 192 pp.

Margalef, R. 1958. Information theory in ecology. General Systems, 3:36-71.

Margalef, R. 1972. Homage to Evelyn Hutchinson, or why there is an upper limit to diversity. Conn. Acord. Sci. Transp., 44:214-235.
Matos, F.D.A.; Amaral, I.L. 1999. Análise ecológica de um hectare em floresta ombrófila densa de terra-firme, estrada da várzea, Amazonas, Brasil. Acta Amazonica, 29:365-379.

Müller-Dombois D.; Ellemberg, H. 1974. Aims and methods for vegetation ecology. John Wiley \& Sons, New York, USA. 547 pp.

Nappo, M.E. 1999. Inventário florístico e estrutural da regeneração natural no sub-bosque de povoamentos bomogêneos de Mimosa scabrella Bentham, implantados em áreas mineradas, em Poços de Caldas, Minas Gerais. Dissertação de Mestrado. UFV. Viçosa, MG. 123 pp.

Odum, E.P. 1983. Ecologia. Rio de Janeiro, RJ: Ed. Guanabara. 434pp.

Pielou, E.C. 1966. Species-diversity and pattern-diversity in the study of ecological sucession. J. Theoret. Biol., 13:131-144.

Poggiani, F. 1989. Estrutura, funcionamento e classificação de florestas: implicação ecológica em florestas plantadas. Documentos Florestais, 3:9-14.

Porto, M.L.; Longhi, H.M.; Citadini, V.; Ramos, R.F.; Mariath, J.E.A. 1976. Levantamento fitossociológico em área de "mata-debaixio", na estação Experimental de Silvicultura Tropical INPA - Manaus - Amazonas. Acta Amazonica, 6:301-318.

Rankin-de-Mérona, J.; Prance, G.T.; Hutchings, R.W.; Silva, M.F.; Rodrigues, W.A. 1992. Preliminary results of a large-scale tree inventory of upland rain forest in the Central Amazon. Acta Amazonica, 22:485-492.

Ribeiro, M.N.G.; Adis, J. 1984. Local rainfall variability - a potencial bias for bioecological studies in the Central Amazon. Acta Amazonica, 14:159-174.

Ribeiro, J.E.L.S, Hopkins, M.J.G.; Vicentini, A.; Sothers, C.A.; Costa, M.A.S.; Brito, J.M.; Souza, M.A.D.; Martins, L.H.P.; Lohmann, L.G.; Assunção, P.A.C.L.; Pereira, E.C.; Silva, C.F.; Mesquita, M.R.; Procópio, L.C. 1999. Flora da Reserva Ducke. Guia de Identificação das Plantas Vasculares de uma Floresta de Terra-firme na Amazônia Central, Instituto Nacional de Pesquisas da Amazônia, Manaus, 793 pp.

Rollet, B. 1978. Arquitetura e crescimento das florestas tropicais. Belém, PA: SUDAM, 22pp.

Tello,J.C.R. 1995. Aspectos fitossociológicos das comunidades vegetais de uma toposseqüência da Reserva Florestal Ducke do INPA. Tese de Doutorado. Instituto Nacional de Pesquisas da Amazônia/ Universidade Federal do Amazonas, Manaus, AM. 335pp.

Viana, V.M.; Tabanez, A.J.A.; Maratinez, J.F.A. 1992. Restauração e manejo de fragmentos florestais. Revista do Instituto Florestal de São Paulo, 4:400-406.

Vieira, G. 1989. Composição florística da regeneração natural 1 ano após diferentes níveis de exploração de uma floresta tropical úmida. Acta Amazonica, 19:401-413.

Volpato, M.M.P. 1994. Regeneração natural de uma floresta secundária no domínio de mata atlântica: uma análise fitossociológico. Dissertação de Mestrado. UFV. Viçosa, MG. 123pp.

\section{RECEBIDO EM 03/10/2003 ACEITO EM 15/01/2005}

